\title{
Least-squares reverse time migration with local Radon-based preconditioning
}

\author{
Gaurav Dutta ${ }^{1}$, Matteo Giboli ${ }^{2}$, Cyril Agut ${ }^{2}$, Paul Williamson ${ }^{2}$, and Gerard T. Schuster ${ }^{1}$
}

\begin{abstract}
Least-squares migration (LSM) can produce images with better balanced amplitudes and fewer artifacts than standard migration. The conventional objective function used for LSM minimizes the L2-norm of the data residual between the predicted and the observed data. However, for field-data applications in which the recorded data are noisy and undersampled, the conventional formulation of LSM fails to provide the desired uplift in the quality of the inverted image. We have developed a leastsquares reverse time migration (LSRTM) method using local Radon-based preconditioning to overcome the low signal-tonoise ratio $(\mathrm{S} / \mathrm{N})$ problem of noisy or severely undersampled data. A high-resolution local Radon transform of the reflectivity
\end{abstract}

is used, and sparseness constraints are imposed on the inverted reflectivity in the local Radon domain. The sparseness constraint is that the inverted reflectivity is sparse in the Radon domain and each location of the subsurface is represented by a limited number of geologic dips. The forward and the inverse mapping of the reflectivity to the local Radon domain and vice versa is done through 3D Fourier-based discrete Radon transform operators. The weights for the preconditioning are chosen to be varying locally based on the relative amplitudes of the local dips or assigned using quantile measures. Numerical tests on synthetic and field data validate the effectiveness of our approach in producing images with good $\mathrm{S} / \mathrm{N}$ and fewer aliasing artifacts when compared with standard RTM or standard LSRTM.

\section{INTRODUCTION}

Least-squares migration (LSM), or linearized inversion (Lailly, 1984), has been shown to produce images with balanced amplitudes, better resolution, and fewer artifacts than standard migration (Schuster, 1993; Nemeth et al., 1999; Duquet et al., 2000; Plessix and Mulder, 2004; Tang, 2009; Dai and Schuster, 2010; Wong et al., 2011; Dutta and Schuster, 2014). Besides a migration of the data residual, every iteration of LSM involves Born modeling to estimate the step length and to update the data residual. The potential of LSM, especially least-squares reverse time migration (LSRTM), to produce images of superior quality than any other conventional migration technique has been wellstudied in recent years.

The problems associated with LSRTM can be broadly grouped into three major categories: (1) inadequate physics taken into account by the modeling and the adjoint equations, (2) errors in the migration velocity model, and (3) the computational cost. For reducing the computational cost, phase-encoded migration (Morton and Ober, 1998; Romero et al., 2000) was proposed, which was later extended to multisource LSRTM by Dai et al. (2010), Schuster et al. (2011), and Dai et al. (2012). They show that by an iterative migration of supergathers, multisource LSRTM can produce more accurate reflectivity images than standard reverse time migration (RTM) and at a fraction of the cost of standard LSRTM. A similar approach is adopted by Boonyasiriwat and Schuster (2010) and Herrmann and Li (2012); they use a combination of randomized dimensionality reduction and divide-and-conquer techniques to decimate the LSM problem as a series of smaller subproblems in which each subproblem involved iterating on a small randomized subset of the data. Herrmann and Li (2012) also combine their approach with compressive sensing (Candes et al., 2006b) and curvelet-domain sparse recovery (Candes et al., 2006a) to mitigate the crosstalk noise.

\footnotetext{
Manuscript received by the Editor 5 March 2016; revised manuscript received 14 September 2016; published online 16 February 2017.

${ }^{1}$ King Abdullah University of Science and Technology, Department of Earth Science and Engineering, Thuwal, Saudi Arabia. E-mail: gaurav.dutta@kaust.edu .sa; gerard.schuster@kaust.edu.sa.

TOTAL Exploration and Production, CSTJF, Pau, France. E-mail: matteo.giboli@total.com; cyril.agut@total.com; paul.williamson@total.com.

(C) 2017 Society of Exploration Geophysicists. All rights reserved.
} 
Besides the computational cost and errors in the migration velocity model, the other major problem related to LSM is insufficient physics taken into account by the modeling and the adjoint equations. This is because the real earth is anisotropic and anelastic and estimation of all the subsurface parameters that have significant effect on wave propagation is not trivial. Limitations in our acquisition capabilities, especially related to data sampling and illumination, also remain an issue. Thus, for field-data applications of LSM, it becomes necessary to incorporate some form of regularization or preconditioning into the least-squares inversion that would allow for a more accurate representation of the subsurface reflectivity and mitigate some of these problems. The inverted image should also be consistent with any prior information that is available for it (Li et al., 2015).

However, in the absence of well logs, getting reliable prior information that can be used as constraints to guide the inversion is not straightforward. A more popular choice is to impose constraints on the quality of the image that is desired. For example, Wang and Sacchi (2007) use a cost function for one-way wave-equation-based LSM with regularization constraints for smoothness along offsetdomain common-image gathers and reflectivity sparseness in depth. Cabrales-Vargas and Marfurt (2013) also formulate a regularized least-squares Kirchhoff migration problem in which they use a penalty function that controls the amount of roughness in common reflection point gathers (CRPGs). They use a three-point mean filter in every CRPG to remove the aliasing artifacts. Total variation regularization-based approaches (Anagaw and Sacchi, 2012; Lin and Lianjie, 2015) have also been used with LSM to obtain images with sharp interfaces and discontinuities.

Another approach is to use a change of basis for the reflectivity using linear sparse transforms or some form of model reparameterization (Harlan, 1995; Fomel and Guitton, 2006). Sparseness constraints can then be imposed on the image in the new domain because the true reflectivity is never sparse in the image domain. The choice of a suitable transform is dependent on (1) perfect reconstruction of the parameters after forward and inverse transforms, (2) compatibility with the conjugate gradient algorithm, (3) efficient computation, and (4) minimal redundancy (Kingsbury, 2001). Miller et al. (2005) use the dual-tree complex wavelet transform as a basis for the reflectivity and demonstrate that such a change of basis leads to a better reduction in noise and migration artifacts, whereas at the same time, the discontinuities are preserved better than standard LSM for very sparse data. Herrmann et al. (2009) and Herrmann and Li (2012) use curvelets, and Dutta $(2015,2017)$ use seislets as basis functions for the reflectivity and show that with sparsity promoting imaging techniques, it is possible to recover high-quality images from undersampled or noisy data.

In this paper, we use a local Radon-based preconditioning approach for LSRTM to overcome some of the problems associated with LSRTM when the recorded data are noisy and the background velocity model is inaccurate. We use the prior information that the inverted reflectivity is sparse in the local Radon domain and each location of the subsurface is represented by a limited number of dips. The LSRTM problem is then posed as an optimization problem that minimizes the L1-norm of the local Radon transform of the image subject to the condition that the data misfit is minimized to an acceptable tolerance level. By the attribute local, we imply that the image generated by a shot is divided into subdomains (or small probes), and the Radon transform is then applied to each of the individual subdomains. The forward mapping to the local Radon domain and its inverse is done through 3D discrete Radon transform operators in the Fourier domain. Using model reparameterization and reweighting, the problem is solved as a preconditioning problem in which the weights are chosen based on the amplitudes of the events in the dip domain. Numerical tests on synthetic and 3D marine field data show that Radon-based preconditioning can produce images with fewer migration artifacts and better signal-to-noise ratio $(\mathrm{S} / \mathrm{N})$ than standard RTM or LSRTM in a few iterations. Even at greater depths at which migration velocity errors accumulate, the preconditioning approach is shown to produce images with better focusing and fewer artifacts than standard RTM and LSRTM.

This paper is organized into five sections. After the Introduction, the second section describes the theory of LSRTM using local Radon-based preconditioning. Numerical results on synthetic and field data are then presented in the third section. The limitations of the proposed method are discussed in the fourth section, and the conclusions are in the last section.

\section{THEORY}

For a regularized L2-norm inverse problem, we seek to find a solution that best explains the observed data and is consistent with the prior knowledge that is available before any observations are made. This can be obtained by minimizing the misfit function over the image space $\mathbf{M}$ given by

$$
\min _{\mathbf{m} \in \mathbf{M}}\left[\phi(\mathbf{m})=\frac{1}{2}\|\mathbf{d}-\mathbf{L m}\|_{2}+\lambda \Re(\mathbf{m})\right],
$$

where $\mathbf{d}$ represents the observed data, $\mathbf{m}$ represents the reflectivity model, and $\mathfrak{K}(\mathbf{m})$ can be any generic discrete regularizer/prior that imposes constraints on the solution $\mathbf{m}$. These constraints can be such that the reflectors in $\mathbf{m}$ should be sharp (the wavelet is assumed to be deconvolved) or $\Re(\mathbf{m})$ can be chosen to be a functional that measures how far $\mathbf{m}$ is from sparsity in some domain in which it is expected to be sparse; $\mathbf{L}$ represents a linear modeling operator and $\lambda>0$ controls the strength of the regularization term. For LSRTM, $\mathbf{L}$ also represents the Born modeling operator.

If $\lambda=0$, equation 1 is equivalent to solving an unconstrained optimization problem in which the emphasis is purely on minimizing the misfit between the predicted and the observed data. For incomplete, undersampled, or noisy data, emphasizing only the data misfit can lead to images that are degraded in quality with iterations because of overfitting the noise. In the case of LSM, errors in the migration velocity model can also lead to defocusing of images with iterations (Dutta et al., 2014). Thus, for real data applications, it is necessary to incorporate some sort of regularization term into the inversion that allows for a more accurate representation of the subsurface reflectivity model.

If $\lambda>0$, equation 1 solves a constrained optimization problem in which the trade-off between the data fit and the regularization term is controlled by the damping factor $\lambda$. This damping parameter can be estimated by trial-and-error, or a more rigorous procedure is to obtain it from L-shaped curves constructed by a log-log plot of the length of the model vector versus the length of the residual vector for different choices of $\lambda$ (Calvetti et al., 2000). However, such an approach is not feasible for industrial-scale 3D seismic inversion problems. 
An alternative approach is to reformulate the problem in equation 1 as

$$
\min _{\mathbf{m} \in \mathbf{M}}\|\mathbf{R} \mathbf{m}\|_{1} \quad \text { subject to }\|\mathbf{d}-\mathbf{L m}\|_{2}<\epsilon,
$$

where $\mathbf{R}$ can be any suitable sparse transform of choice such that the magnitude-sorted coefficients of the vector $\mathbf{R m}$ are of rapid decay (Herrmann et al., 2008; Fomel and Liu, 2010). The optimization problem in equation 2 minimizes the L1-norm of the model in the transformed domain, and $\epsilon$ is the tolerance level to which the L2-norm data misfit is minimized.

In our case, we take $\mathbf{R}$ to be the local Radon transform of the different subdomains in an image that is computed using a 3D Fourier-based discrete Radon transform algorithm (see Appendix A). We also use the prior information that each location of the subsurface is represented by a limited number of geologic dips and is thus sparse in the local Radon domain.

The intuition behind choosing the local Radon domain to promote sparsity for planar events is illustrated in Figure 1. It can be seen from this figure that for the true reflectivity model in Figure 1a, a sparse representation of the model can be obtained in the local Radon domain in Figure 1b. However, for the migration image in Figure 1c that is obtained from aliased or noisy data, the coefficients in the local Radon domain are no longer sparse, as shown in Figure 1d. Thus, in the context of LSRTM, we seek to invert for a reflectivity image that minimizes the L2-norm data residual in equation 2 and at the same time, the coefficients of the inverted image are sparse in the local Radon domain.

For computational efficiency, we use an iteratively reweighted least-squares algorithm (IRLS) (Holland and Welsch, 1977; Trad et al., 2003; Daubechies et al., 2010). Using a weighting matrix $\mathbf{W}$, equation 2 can be rewritten for a single iteration as

$\min _{\mathbf{m} \in \mathbf{M}}\|\mathbf{W R m}\|_{2} \quad$ subject to $\|\mathbf{d}-\mathbf{L m}\|_{2}<\epsilon$,

where WRm is a weighted linear Radon transform operation that makes the model vector $\mathbf{m}$ sparse in the transformed domain after the weighting. In our work, we have chosen the weights as a function of the amplitudes of the local dip events; i.e., the diagonal elements of $\mathbf{W}$ are given by

$$
[\mathbf{W}]_{i i}=\frac{1}{\sqrt{\left|\hat{m}_{i}\right|}+\xi}, \quad \hat{\mathbf{m}}=\mathbf{R m}
$$

where the weights are computed by using the solution of the previous iteration and $\xi$ is a threshold parameter used to avoid division by zero. For such a weighting matrix $\mathbf{W}$, it can be shown that

$$
\begin{aligned}
\|\mathbf{R m}\|_{1} & =\|\hat{\mathbf{m}}\|_{1}=\sum_{i}\left|\hat{m}_{i}\right|=\hat{\mathbf{m}}^{T} \mathbf{W}^{T} \mathbf{W} \hat{\mathbf{m}} \\
& =\|\mathbf{W} \hat{\mathbf{m}}\|_{2}=\|\mathbf{W} \mathbf{R m}\|_{2}
\end{aligned}
$$

Alternatively, the weighting matrix $\mathbf{W}$ can also be constructed using some quantile-based measure such that beyond a certain threshold level, the weaker dips corresponding to the noise are down-weighted.

The normal equations corresponding to the misfit function in equation 3 are given by

$$
\left(\lambda \mathbf{R}^{\mathbf{T}} \mathbf{W}^{\mathbf{T}} \mathbf{W} \mathbf{R}+\mathbf{L}^{\mathbf{T}} \mathbf{L}\right) \mathbf{m}=\mathbf{L}^{\mathbf{T}} \mathbf{d},
$$

which can be solved by combining the IRLS algorithm with conjugate-gradient-based least-squares inversion (Scales et al., 1988; Nocedal and Wright, 1999). The solution $\mathbf{m}$ for the normal equation 6 spans over the Krylov subspace (Saad, 2003),

$$
\begin{aligned}
\kappa^{\mathbf{L}^{\mathbf{T}} \mathbf{L}+\lambda \mathbf{R}^{\mathbf{T}} \mathbf{W}^{\mathbf{T}} \mathbf{W R}}= & \operatorname{span}\left\{\mathbf{L}^{\mathbf{T}} \mathbf{d},\left(\mathbf{L}^{\mathbf{T}} \mathbf{L}+\lambda \mathbf{R}^{\mathbf{T}} \mathbf{W}^{\mathbf{T}} \mathbf{W} \mathbf{R}\right) \mathbf{L}^{\mathbf{T}} \mathbf{d}, \ldots,\right. \\
& \left.\left(\mathbf{L}^{\mathbf{T}} \mathbf{L}+\lambda \mathbf{R}^{\mathbf{T}} \mathbf{W}^{\mathbf{T}} \mathbf{W} \mathbf{R}\right)^{i_{\max }-1} \mathbf{L}^{\mathbf{T}} \mathbf{d}\right\} \subset \mathbf{M},
\end{aligned}
$$

where $i_{\max }$ is the limit of the Krylov subspace or the number of iterations carried out.

The solutions in equation 7 are controlled by the damping parameter $\lambda$ and $i_{\max }$. Because the optimum estimation of $\lambda$ is not trivial, a model reparameterization or preconditioning approach is convenient to estimate the reflectivity $\mathbf{m}$ (Harlan, 1995; Fomel and Guitton, 2006). If $\mathbf{W}$ and $\mathbf{R}$ are invertible, we can write

$$
\mathbf{p}=\mathbf{W} \mathbf{R m}, \quad \mathbf{m}=\mathbf{R}^{-1} \mathbf{W}^{-1} \mathbf{p},
$$

such that equation 3 gives

$$
\min _{\mathbf{p} \in \mathbf{W R M}}\|\mathbf{p}\|_{2} \quad \text { subject to }\left\|\mathbf{d}-\mathbf{L R}^{-\mathbf{1}} \mathbf{W}^{-\mathbf{1}} \mathbf{p}\right\|_{2}<\epsilon .
$$
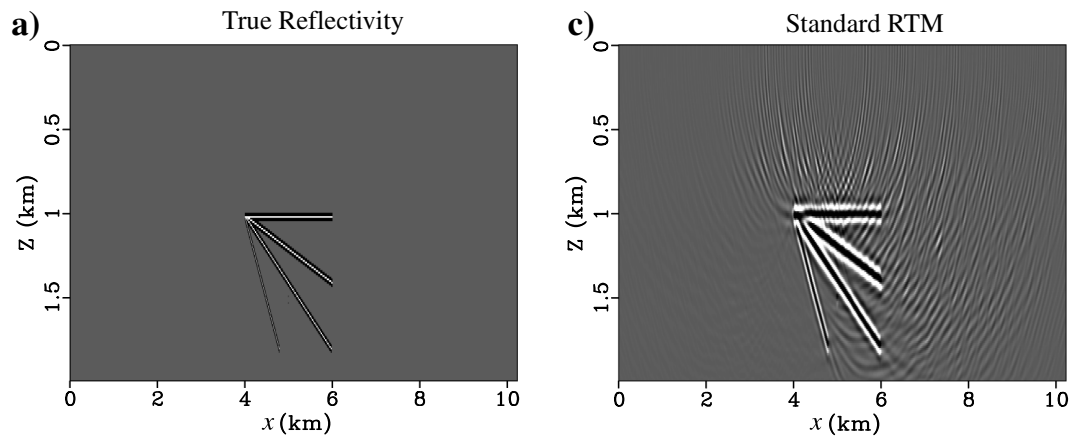

b) Local Radon transform of panel (a)
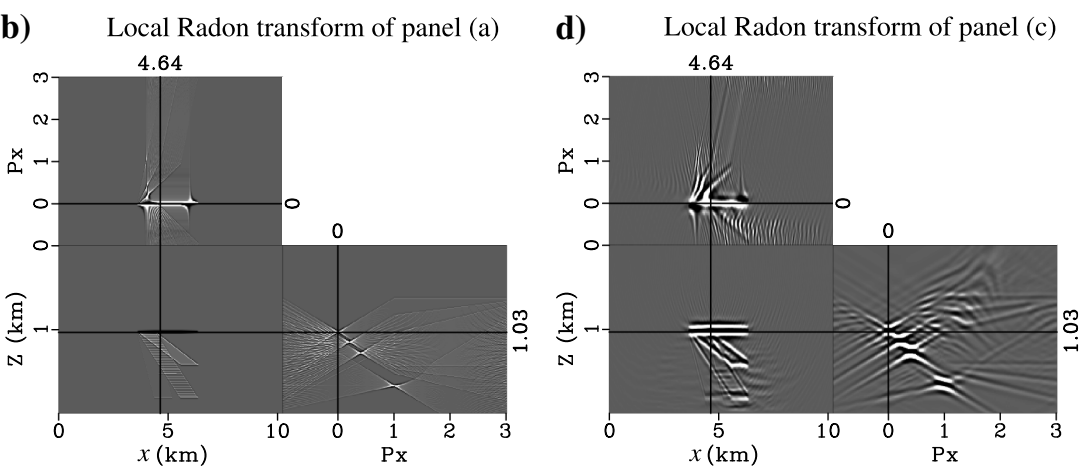

Figure 1. (a) True reflectivity model, (b) local Radon transform of (a), (c) standard RTM image obtained from aliased data, and (d) local Radon transform of (c). 
The normal equations corresponding to the misfit function in equation 9 are now given by

$$
\left(\lambda \mathbf{I}+\mathbf{W}^{-\mathbf{T}} \mathbf{R}^{-\mathbf{T}} \mathbf{L}^{\mathbf{T}} \mathbf{L R}^{-1} \mathbf{W}^{-1}\right) \mathbf{p}=\mathbf{W}^{-\mathbf{T}} \mathbf{R}^{-\mathbf{T}} \mathbf{L}^{\mathbf{T}} \mathbf{d} .
$$

Setting $\lambda=0$ in equation 10 , we get the new normal equations

$$
\left(\mathbf{W}^{-\mathbf{T}} \mathbf{R}^{-\mathbf{T}} \mathbf{L}^{\mathbf{T}} \mathbf{L R}^{-1} \mathbf{W}^{-1}\right) \mathbf{p}=\mathbf{W}^{-\mathbf{T}} \mathbf{R}^{-\mathbf{T}} \mathbf{L}^{\mathbf{T}} \mathbf{d},
$$

where the solution $\mathbf{p}$ for the normal equations in equation 11 spans over the Krylov subspace

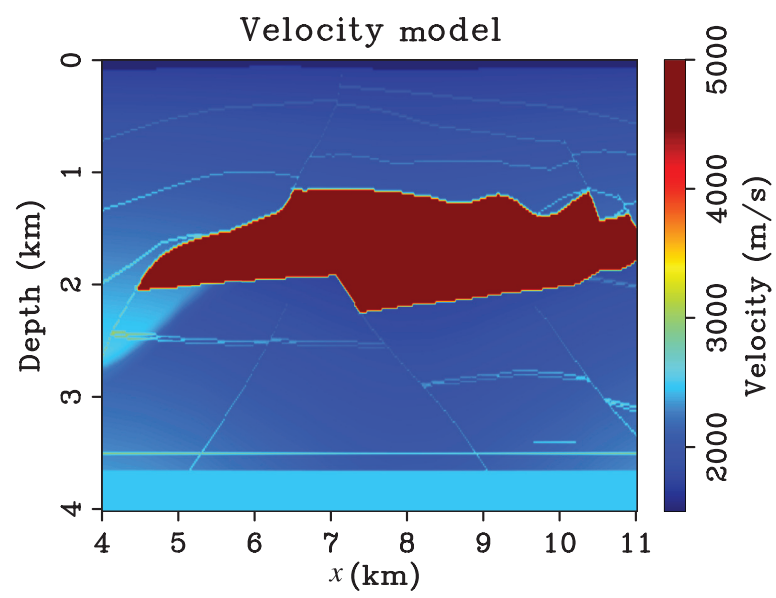

Figure 2. A 2D slice of the 3D SEG/EAGE salt model.
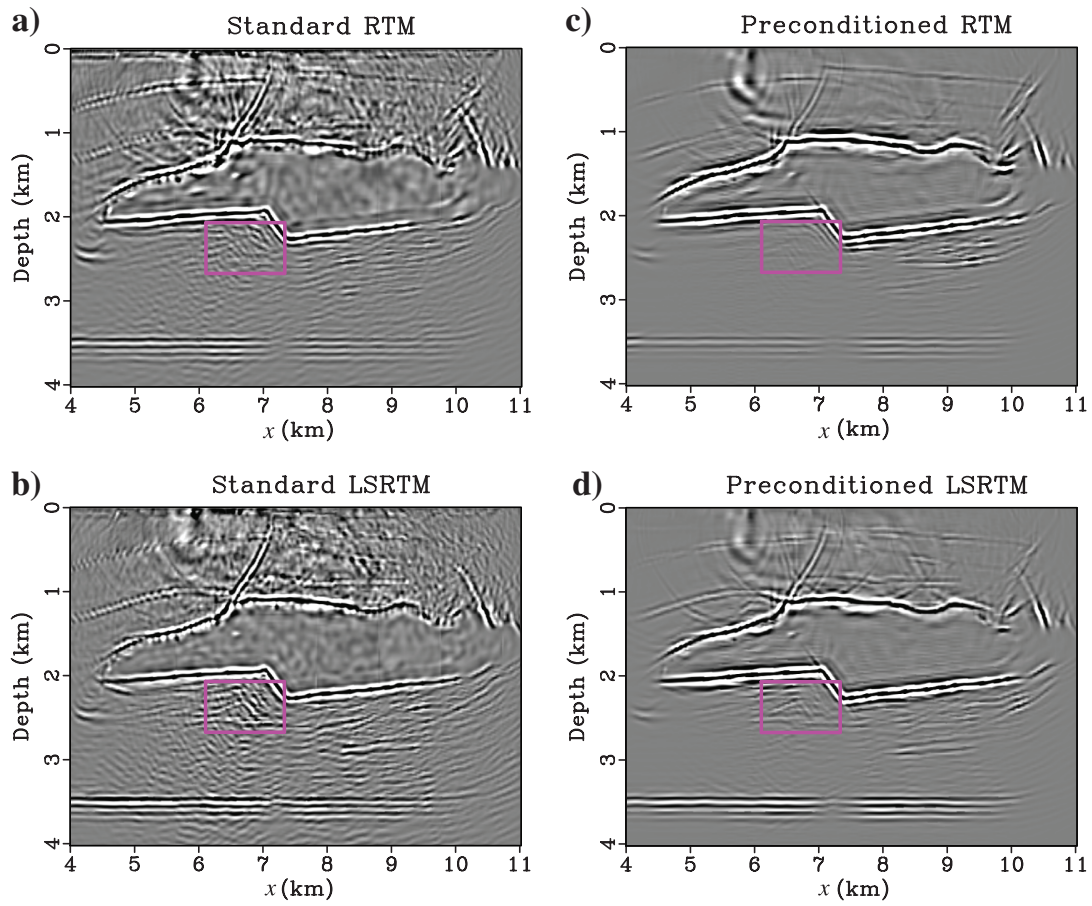

Figure 3. Comparison of images from (a) standard isotropic RTM, (b) standard isotropic LSRTM after five iterations, (c) preconditioned isotropic RTM, and (d) preconditioned isotropic LSRTM after five iterations.

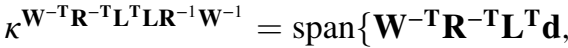

$$
\begin{aligned}
& \left(\mathbf{W}^{-\mathbf{T}} \mathbf{R}^{-\mathbf{T}} \mathbf{L}^{\mathbf{T}} \mathbf{L} \mathbf{R}^{-1} \mathbf{W}^{-1}\right) \mathbf{W}^{-\mathbf{T}} \mathbf{R}^{-\mathbf{T}} \mathbf{L}^{\mathbf{T}} \mathbf{d}, \\
& \ldots,\left(\mathbf{W}^{-\mathbf{T}} \mathbf{R}^{-\mathbf{T}} \mathbf{L}^{\mathbf{T}} \mathbf{L} \mathbf{R}^{-1} \mathbf{W}^{-1}\right)^{\mathbf{i}_{\max }-1} \\
& \left.\mathbf{W}^{-\mathbf{T}} \mathbf{R}^{-\mathbf{T}} \mathbf{L}^{\mathbf{T}} \mathbf{d}\right\} \subset \mathbf{W R M} \text {. }
\end{aligned}
$$

On comparing the Krylov spaces in equations 7 and 12, it can be seen that sparseness constraints are imposed on the solutions in equation 12 by the action of the Radon transform and the weighting operators and the number of iterations of the conjugate gradient algorithm. In contrast, the constraints in equation 7 are controlled by the parameter $\lambda$. We adopt the approach of Trad et al. (2003) and Ibrahim and Sacchi (2014) and set $\lambda=0$ in equation 10, and we let the number of internal iterations in the conjugate gradient algorithm play the role of the regularizer. Here, the iterations for the linear conjugate gradient solver are referred to as the internal iterations, whereas the external iterations refer to updating the weights. Even though the system of equations is partially solved at every external iteration because the conjugate gradient algorithm is stopped before the solution is complete, the total matrix operator resulting from the preconditioning is diagonalized enough that one can avoid using a damping factor for the smallest eigenvalues.

Such a preconditioning approach is useful because it provides a way of incorporating the prior information (that the inverted reflectivity should be sparse in the local Radon domain) directly into the forward operator (Harlan, 1995; Fomel and Guitton, 2006). Otherwise, the solution would require a large number of iterations in the counterpart regularized problem. A large number of iterations will be required before the small eigenvalues become dominant and the corresponding

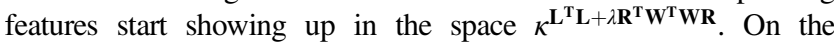
other hand, the vectors in the Krylov space

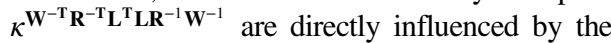
transformation and the weighting operators acting on the gradients at every iteration. Thus, the images from the very first iteration are expected to be sparser in the local Radon domain and cleaner with good $\mathrm{S} / \mathrm{N}$ and fewer migration artifacts in the image domain for the preconditioned problem in equation 9 than with the regularized problem in equation 1 .

\section{NUMERICAL RESULTS}

The effectiveness of LSRTM using Radon-based preconditioning is now demonstrated with synthetic and field data examples. The synthetic example is on the 3D SEG/EAGE salt model, whereas the field data example is on a 3D marine data set.

In the synthetic example, the observed data are generated by an $\mathrm{O}(2,8)$ time-space-domain solution of the constant-density second-order acousticwave equation without a free surface. A Ricker wavelet with a $15 \mathrm{~Hz}$ peak frequency is chosen as the source wavelet. The data are then migrated using RTM, LSRTM, preconditioned RTM, and preconditioned LSRTM. Here, RTM and preconditioned RTM refer to the first internal iteration of standard LSRTM and our proposed preconditioned LSRTM, respectively. Source-side illumina- 
tion compensation (Plessix and Mulder, 2004) is used as an additional preconditioner for standard and preconditioned LSRTM. All the images have also been filtered using a classic band-pass filter using the same low-cut and high-cut frequencies.

We ran 10 internal iterations for the synthetic data set, whereas only five internal iterations are carried out for the $3 \mathrm{D}$ field data set. In all the numerical examples, the Radon transform is applied shot by shot, i.e., using a prestack LSRTM algorithm (Dai and Schuster, 2013; Duprat and Baina, 2016). An image generated by a shot is divided into subdomains and the Radon transform is applied to each of the individual subdomains. The transform is applied using three sequential modes in which each of the mode scans dips from $-45^{\circ}$ to $+45^{\circ}$ in the $x$-, $y$-, and $z$-planes at every internal iteration using the Radon transform formulation given in equations A-1, A-4, and A-5, respectively. Thus, using this approach, we scan for all possible geologic dips in the dip space of this image.

In all the numerical examples, the full length of the fast axis ( $z$ in mode 1, $x$ in mode 2, and $y$ in mode 3 ) and an extension of $40 \times 40$ samples in the two other directions is chosen as the size of a subdomain. We do not compute as many Radon transforms as the number of common-depth points (CDPs)/pixels in the image, but we allow a shift between the center of successive subdomains by four samples. Thus, the different subdomains have an overlap.

The size of a subdomain is chosen based on the number of equally spaced slopes that should be considered in the range $-45^{\circ}$ to $+45^{\circ}$ in each of the three modes. Equation A-11 describes the duality in the number of samples in a subdomain and the number of slopes considered in the Radon transform. For a discrete image $I$ of size $n \times n \times n, n$ equally spaced slopes are considered in each of the three modes in the range $-45^{\circ}$ to $+45^{\circ}$.

\section{Synthetic example}

We first demonstrate the effect of using Radon-based preconditioned LSRTM on the 3D SEG/EAGE salt model. There are only 45 shots recorded on a $5 \times 9$ source grid with a $960 \mathrm{~m}$ shot and shot-line separation. Each shot is recorded by receivers on a $201 \times 201$ receiver grid with a $50 \mathrm{~m}$ spacing between the receivers. The observed data are recorded using the true velocity model (a 2D slice is shown in Figure 2) for approximately $8 \mathrm{~s}$ with a $4 \mathrm{~ms}$ sampling rate. For migration, we smooth the true slowness model using a triangle smoothing filter with a smoothing radius of $50 \mathrm{~m}$ in each of the three directions.

Figure 3a-3d compares 2D slices of the images from standard RTM, standard LSRTM, preconditioned RTM, and preconditioned LSRTM, respectively. The RTM image in Figure 3a suffers from very strong backscattering noise because of the presence of the salt body. The slices show strong acquisition footprints because of the sparse acquisition geometry, and the reflector amplitudes are also very weak. The image contains significant high-frequency noise because of using severely undersampled data. The LSRTM image in Figure 3b shows some improvements over the standard RTM image. The reflector amplitudes are better balanced, and the acquisition footprints are mitigated. However, the aliasing noise is still prominent and is severe below the salt body. The preconditioned LSRTM image, shown in Figure 3d, is free from aliasing noise, and the subsalt images are much cleaner when compared with the standard RTM and LSRTM images. The preconditioned images also have a much better $\mathrm{S} / \mathrm{N}$ than the standard RTM and LSRTM images.

\section{D marine data example}

Preconditioned LSRTM is now applied to a 3D marine data set from offshore West Africa. The acquisition is broadband with a shot interval of $18.75 \mathrm{~m}$ (flip flop) and a source center separation of $25 \mathrm{~m}$. The receiver spread has 12 cables with an active length of $6 \mathrm{~km}$. There are 480 receiver groups with a group interval of $12.5 \mathrm{~m}$. The sampling interval is $2 \mathrm{~ms}$ with a total recording time of $8 \mathrm{~s}$. Before tomography and migration, standard processing steps such as swell noise attenuation, linear noise attenuation, and
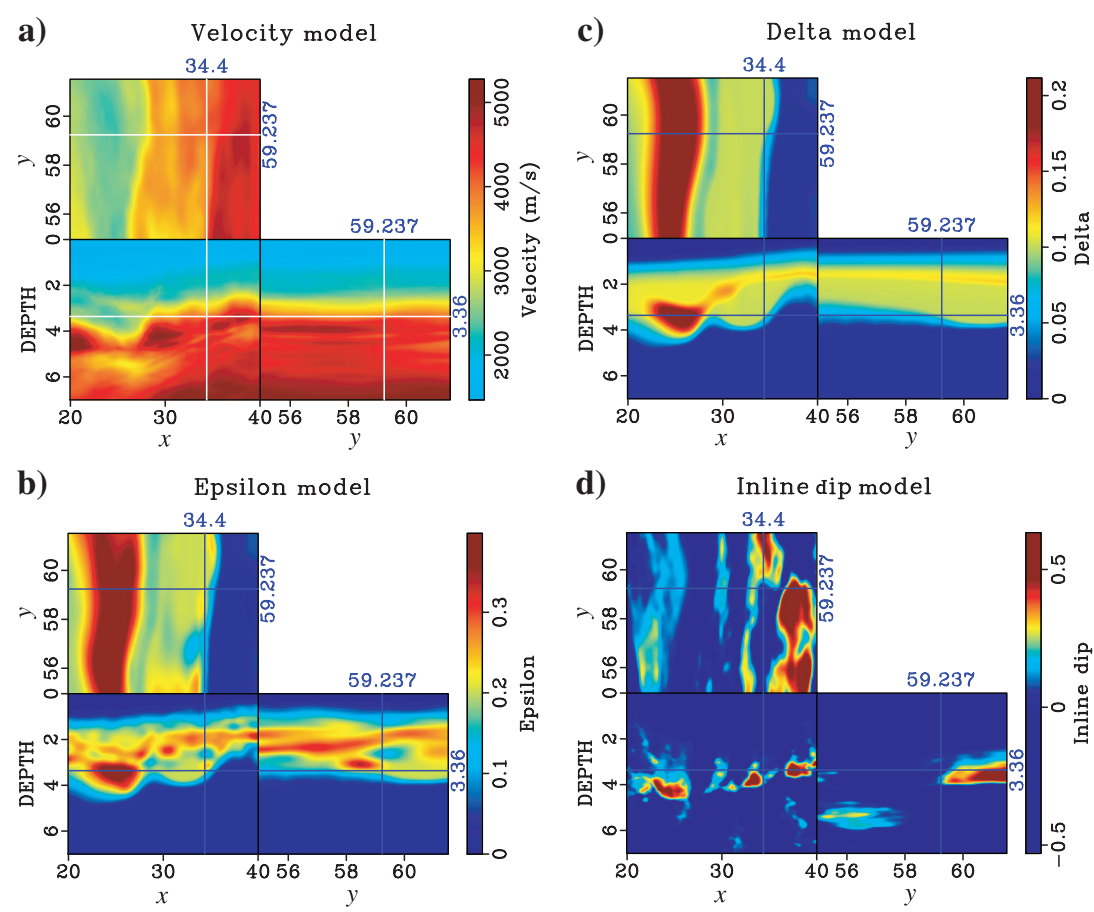

d)
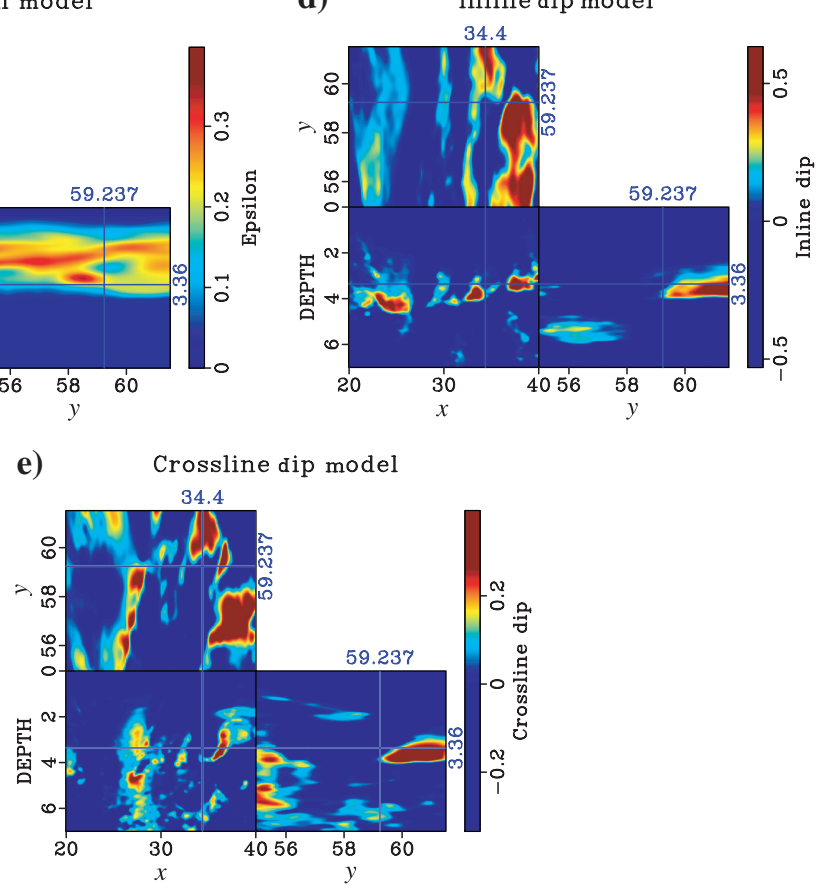

Figure 4. Background (a) velocity, (b) epsilon, (c) delta, (d) inline dip, and (e) crossline dip models used for migration. 
receiver deghosting are carried out. Three-dimensional SRME and Radon demultiple are also used to remove the free-surface multiples.

The background velocity, epsilon, delta, inline dip, and crossline dip models are shown in Figure $4 \mathrm{a}-4 \mathrm{e}$, respectively. The velocity model is obtained using ray-based tomography and full-waveform inversion, whereas ray-based tomography is used to obtain the epsilon model. The delta and dip models are constructed using information from wells in the study area.

For migration, there are 10,241 shots used and a band-pass filter of 3-5-25-30 Hz is applied to the traces. A zero-phase Ricker wavelet band-pass filtered in the same frequency range is used as the source wavelet for LSRTM. The standard tilted transversely isotropic (TTI) RTM and TTI LSRTM images are shown in Figures $5 \mathrm{a}$ and $6 \mathrm{a}$, respectively. It is evident from these images that standard LSRTM improves the image quality (in terms of improved resolution and balanced amplitudes) in the shallow parts. However, the image quality of the carbonate turtlebacks at depths of $3-5 \mathrm{~km}$, shown in Figure 7a and 7b, is degraded in the standard RTM and the standard LSRTM images. We suspect that inaccuracies in the background models or presence of noise in the data might have lead to such degradation. The crossline sections of these images, shown in

a)

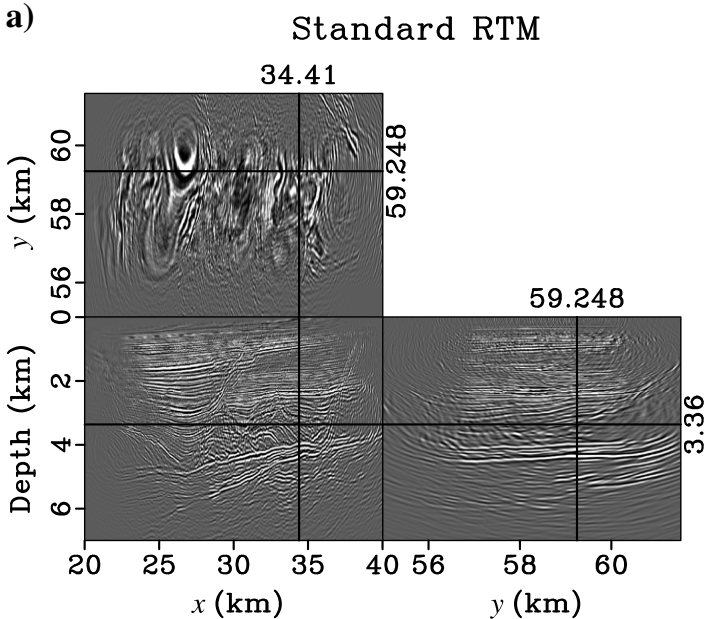

b)

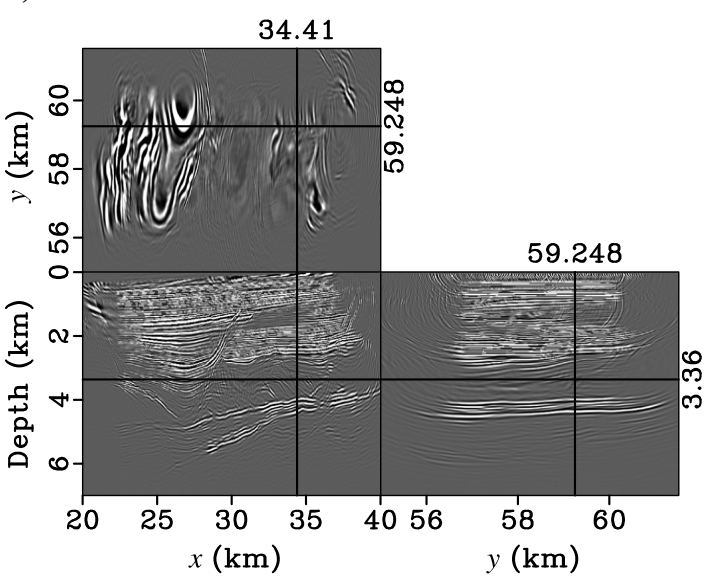

Figure 5. Comparison of images from (a) standard TTI RTM and (b) preconditioned TTI RTM.
Figure $8 \mathrm{a}$ and $8 \mathrm{~b}$, also suffer from strong aliasing artifacts and migration smiles due to large aperture.

The preconditioned TTI RTM and TTI LSRTM images are shown in Figures $5 \mathrm{~b}$ and $6 \mathrm{~b}$, respectively. Magnified views of these images in the inline and crossline directions are shown in Figure $7 \mathrm{c}$ and $7 \mathrm{~d}$ and Figure $8 \mathrm{c}$ and $8 \mathrm{~d}$, respectively. It is evident from these images that the turtlebacks are imaged with an improved $\mathrm{S} / \mathrm{N}$, and they can be clearly delineated. The aliasing noise has also been significantly mitigated in the crossline images.

It is also interesting to note that some of the events that are focused in the standard RTM image at depths of approximately $4.5-5 \mathrm{~km}$, shown in the right black box in Figure 7a, got defocused in the standard LSRTM image in Figure 7b. We suspect that errors in the migration velocity model might have led to such degradation in the standard LSRTM image. Dutta et al. (2014) show that the quality of the inverted image from LSRTM is very sensitive to errors in the velocity model. They also show that at deeper depths at which migration velocity errors accumulate, the quality of the inverted image from LSRTM becomes worse when compared with the image at shallow depths. Their observation is also consistent

a)

Standard LSRTM

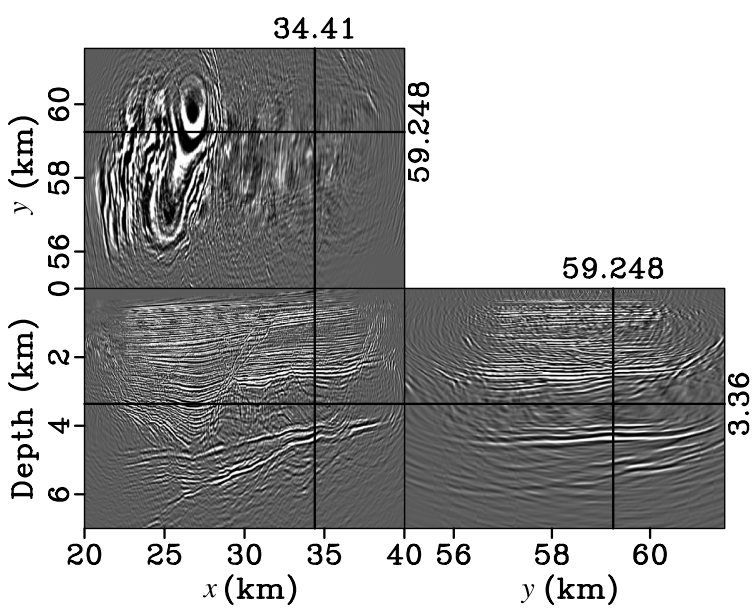

b) Preconditioned LSRTM

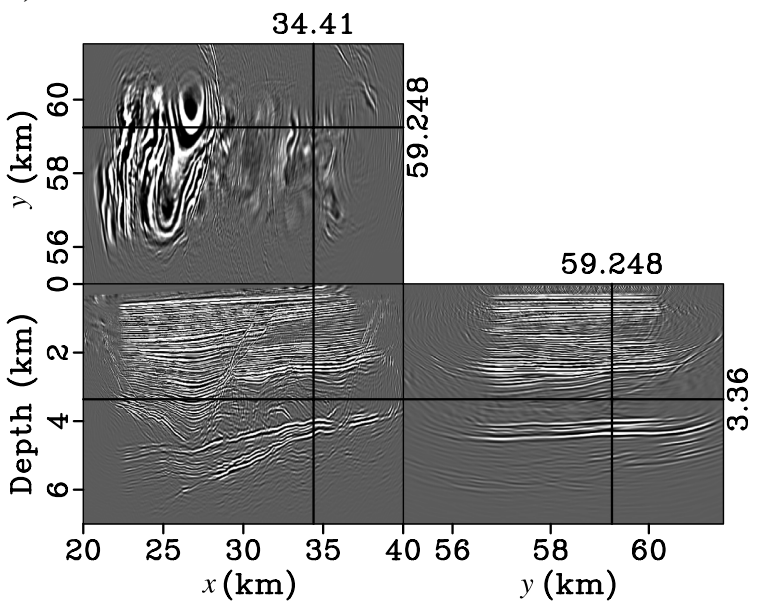

Figure 6. Comparison of images from (a) standard TTI LSRTM and (b) preconditioned TTI LSRTM after five least-squares iterations. 
with what we observe in our case in which the quality of the standard LSRTM image became worse with depth. However, these deeper events are better focused and have fewer artifacts in the preconditioned LSRTM image in Figure 7d.

\section{DISCUSSION}

In our work, we used the prior information that the inverted image is sparse in the local Radon domain. If a genuine low-amplitude event is rejected at the first iteration while imposing sparsity in the local Radon domain, that event will be relatively stronger in the gradient of the second iteration. This is because the predicted data from that event have not been explained in the first iteration and the residual for the next iteration will be dominated by this event. Because the preconditioning operators act on the gradient at every iteration, a strong event in the gradient will have a strong dip signature. Thus, the image updates for that iteration will be dominated by such an event. The final inverted images from the least-squares iterations will have high- and low-amplitude reflectors. This can also be seen in our synthetic and field data examples.

The proposed Radon-based preconditioning approach provides significant uplift in the quality of the inverted images when the desired features are planar and the data used for migration have been carefully preprocessed such that free-surface multiples or internal multiples are eliminated. However, the presence of free-surface or internal multiples in the data will lead to false reflection events in the image, which are not discriminated against by our approach. For example, the blue boxes in Figure 3 highlight the effect of internal multiples below the salt. Unless a very large number of least-squares iterations is carried out, these events will still be quite strong in the image and they will have strong amplitude signatures in the local Radon domain. Filtering out these events in the extended domain is not trivial.

To illustrate our point regarding the effect of free-surface multiples, the synthetic example shown earlier is repeated again. However, we now model the observed data using a free surface. The standard RTM and LSRTM images are shown in Figure 9a and 9b, respectively. The effect of free-surface multiples and internal multiples is evident, especially below the subsalt areas. The preconditioned RTM and LSRTM images, shown in Figure 9c and 9d, are cleaner above the salt, but they have many false events below the salt. This can be verified by comparing these images with the true velocity model in Figure 2. However, we believe that this is not a serious issue because for field data applications a)
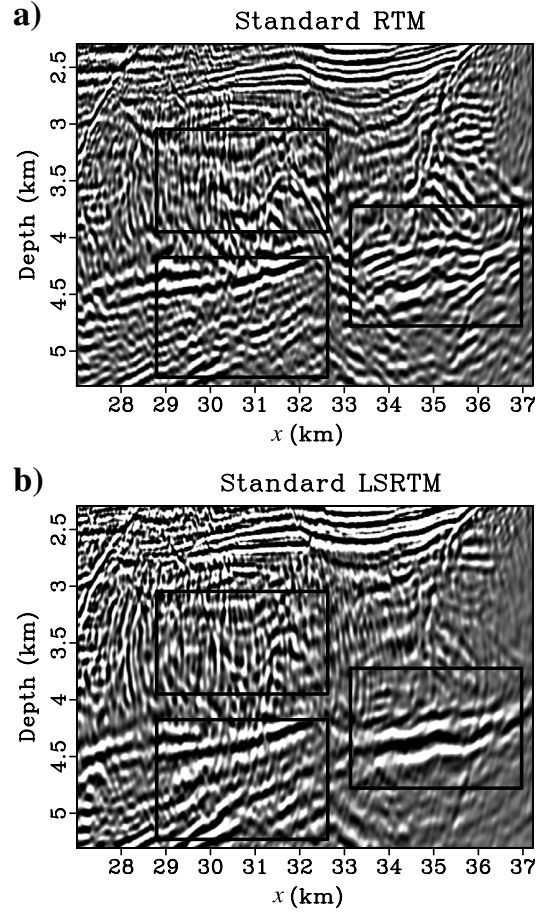

c)

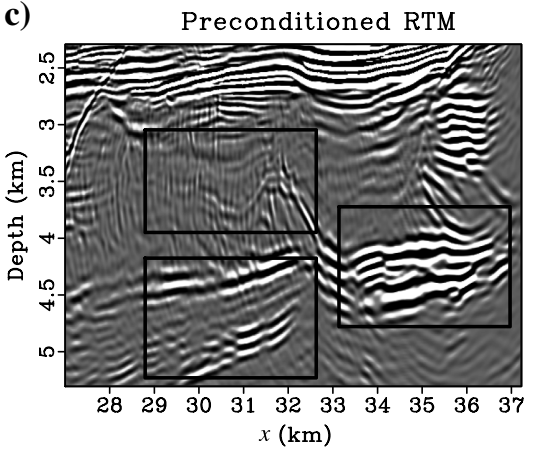

d)

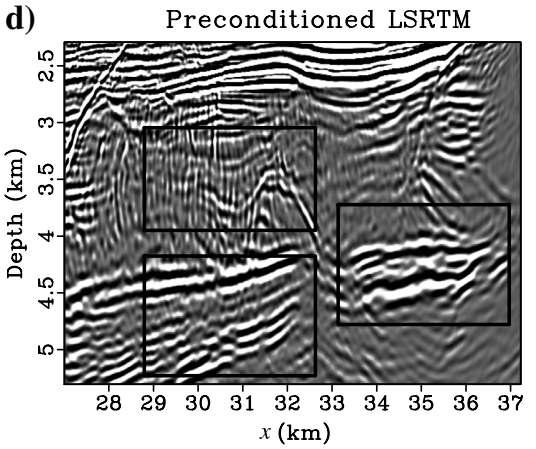

Figure 7. Comparison of magnified views of the images in the inline direction from (a) standard TTI RTM, (b) standard TTI LSRTM, (c) preconditioned TTI RTM, and (d) preconditioned TTI LSRTM. The black boxes indicate the areas where improvements in imaging can be seen from the Radon-based preconditioning.

a)

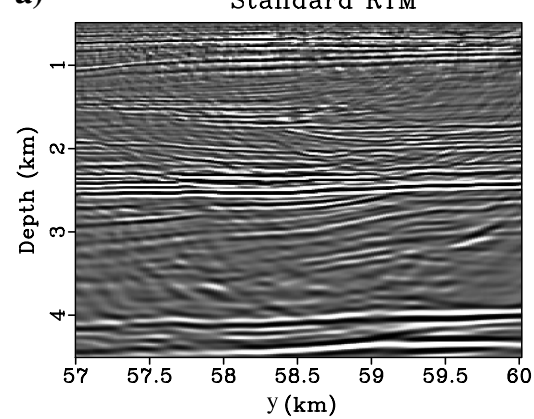

b)

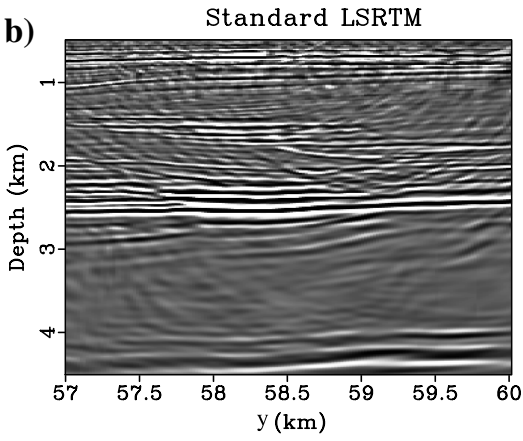

c)
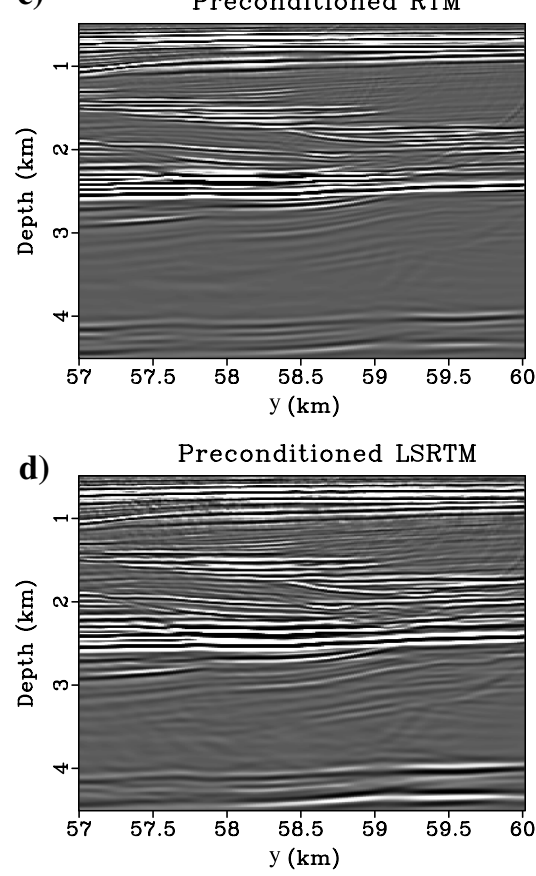

Figure 8. Comparison of magnified views of the images in the crossline direction from (a) standard TTI RTM, (b) standard TTI LSRTM, (c) preconditioned TTI RTM, and (d) preconditioned TTI LSRTM. 

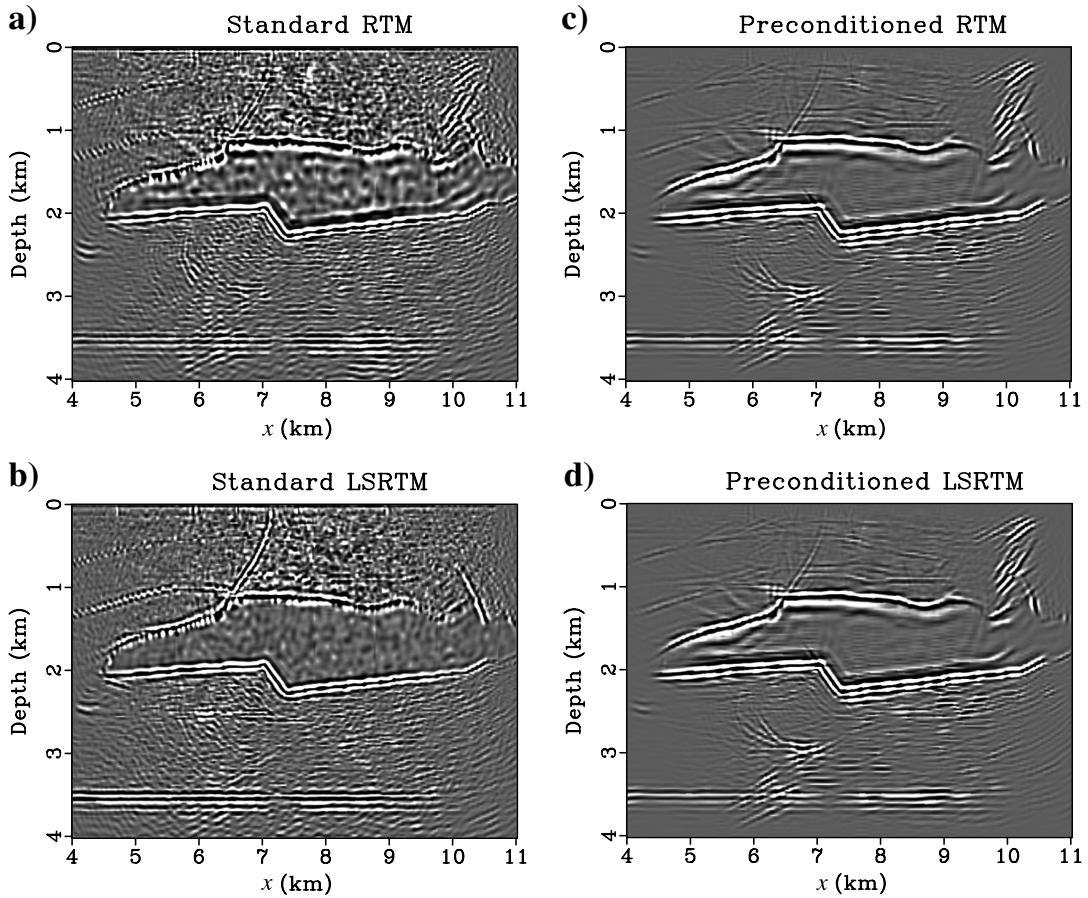

Figure 9. Comparison of images from (a) standard isotropic RTM, (b) standard isotropic LSRTM after five least-squares iterations, (c) preconditioned isotropic RTM, and (d) preconditioned isotropic LSRTM after five least-squares iterations. The recorded data in this case have free-surface multiples.

of LSRTM, careful attention will be paid to the demultiple processing.

Another disadvantage of this method is the additional computational cost incurred during the preconditioning. Solving the normal equations in equation 11 involves storing an extended model to memory at every iteration. For a 2D image $m(x, z)$, a local Radon transform of it will lead to an extended image $\hat{m}\left(x, z, p_{x}\right)$, whereas for a 3D image $m(x, y, z)$, the transformation will lead to a 5D volume $\hat{m}\left(x, y, z, p_{x}, p_{y}\right)$. Here, $p_{x}$ and $p_{y}$ are the local dip angles in the image in the $x$ - and $y$-directions, respectively. The extended models require the computational nodes to have sufficient local memory to avoid using I/O from the disk. Also, there is an additional computational overhead because of the forward and inverse Radon transforms. This cost will vary depending on the size of the model. For our 3D field data example, each iteration of preconditioned LSRTM is roughly three to four times more expensive than standard LSRTM.

\section{CONCLUSION}

A local Radon-based preconditioning approach is presented for LSRTM that mitigates the problems of low S/N data and aliasing. During the least-squares iterations, the image generated by a shot is divided into subdomains and the Radon transform is then applied to each of the individual subdomains. Sparseness constraints are imposed on the reflectivity in the local Radon domain, and the forward and the inverse mapping of the reflectivity to the local Radon domain are done using 3D Fourier-based Radon operators. Numerical tests on synthetic and 3D marine data sets show that if the recorded data are noisy or undersampled, the standard RTM and LSRTM images suffer from poor $\mathrm{S} / \mathrm{N}$ and strong aliasing artifacts. The Radon-based preconditioning approach is shown to produce reliable images with good S/N and fewer artifacts than standard RTM or standard LSRTM at shallow and deeper depths. A disadvantage of this method is that it is quite sensitive to the presence of free-surface multiples or internal multiples in the data. This is because the false reflection events from these multiples have strong local dip amplitudes and as such, they are difficult to filter out. For field data applications, it is important to eliminate these multiples from the data before the proposed preconditioned LSRTM algorithm is applied. Investigating the selection of an appropriate weighting strategy to take into account the effect of multiples is a topic of future research.

\section{ACKNOWLEDGMENTS}

G. Dutta would like to thank King Abdullah University of Science and Technology (KAUST) for funding his graduate studies. The authors are grateful to TOTAL E\&P France for permission to publish this work. We also thank the associate editor K. Innanen and the three anonymous reviewers for their helpful comments and suggestions. The plots for this paper have been prepared using the Madagascar open-source software package.

\section{APPENDIX A}

\section{D FOURIER-BASED DISCRETE RADON TRANSFORM}

The implementation of the 3D Fourier-based discrete Radon transform used in our work is based on the paper by Averbuch and Shkolnisky (2003). The key steps in the computation of the 3D transform, as discussed by Averbuch and Shkolnisky (2003), are shown in this appendix.

For an $x$-plane $x=s_{1} y+s_{2} z+t$ in $\mathbb{R}^{3}$ with slopes $s_{1}$ and $s_{2}$ and intercept $t$, the Radon transform $R_{1} I$ of a discrete image $I$, of size $n \times n \times n$ is given by

$$
\begin{aligned}
R_{1} I\left(s_{1}, s_{2}, t\right) & \triangleq \operatorname{Radon}\left(I, x=s_{1} y+s_{2} z+t\right) \\
& =\sum_{v=-n / 2}^{n / 2-1} \sum_{w=-n / 2}^{n / 2-1} \tilde{I}^{1}\left(s_{1} v+s_{2} w+t, v, w\right),(\mathrm{A}-1)
\end{aligned}
$$

where

$$
\begin{aligned}
\tilde{I}^{1}(x, v, w)= & \sum_{u=-n / 2}^{n / 2-1} I(u, v, w) D_{m}(x-u), \\
& v, w \in\left\{-\frac{n}{2}, \ldots, \frac{n}{2}-1\right\}, \quad x \in \mathbb{R} .
\end{aligned}
$$

Here, $D_{m}$ is the Dirichlet kernel given by 


$$
D_{m}=\frac{\sin (\pi t)}{m \sin (\pi t / m)}, \quad m=3 n+1
$$

where $m$ is the length of the kernel. The length of the Dirichlet kernel in equation A-3 is important to avoid wraparound while summing over straight planes using equation A-1. The wraparound occurs due to the periodic nature of the interpolation kernel $D_{m}$. This can be avoided by padding $I$ with zeros so that the wraparound occurs over the zeros of the padding and not over the true samples of $I$. By geometrically allowing slopes of up to $45^{\circ}$ in each direction, i.e., $\left|s_{1}\right| \leq 1$ and $\left|s_{2}\right| \leq 1$, and the intercept $t$ to be in the interval $-3 n / 2 \leq t \leq 3 n / 2$, the padding size needed along the $z$-axis is $2 n+1$. Thus, the trigonometric interpolation takes places over $(2 n+1)+n=3 n+1$ samples, which is the length of the Dirichlet kernel $D_{m}$ in equation A-3.

Similarly for the $y$-and $z$-planes, the Radon transform is given by

$$
\begin{aligned}
R_{2} I\left(s_{1}, s_{2}, t\right) & \triangleq \operatorname{Radon}\left(I, y=s_{1} x+s_{2} z+t\right) \\
& =\sum_{u=-n / 2}^{n / 2-1} \sum_{w=-n / 2}^{n / 2-1} \tilde{I}^{2}\left(u, s_{1} u+s_{2} w+t, w\right),
\end{aligned}
$$

$$
\begin{aligned}
R_{3} I\left(s_{1}, s_{2}, t\right) & \triangleq \operatorname{Radon}\left(I, z=s_{1} x+s_{2} y+t\right) \\
& =\sum_{u=-n / 2}^{n / 2-1} \sum_{v=-n / 2}^{n / 2-1} \tilde{I}^{3}\left(u, v, s_{1} u+s_{2} v+t\right),
\end{aligned}
$$

where

$$
\begin{aligned}
\tilde{I}^{2}(u, y, w)= & \sum_{v=-n / 2}^{n / 2-1} I(u, v, w) D_{m}(y-v), \\
& u, w \in\left\{-\frac{n}{2}, \ldots, \frac{n}{2}-1\right\}, \quad y \in \mathbb{R}, \\
\tilde{I}^{3}(u, v, z)= & \sum_{w=-n / 2}^{n / 2-1} I(u, v, w) D_{m}(z-w), \\
& u, v \in\left\{-\frac{n}{2}, \ldots, \frac{n}{2}-1\right\}, \quad z \in \mathbb{R} .
\end{aligned}
$$

Using the Fourier slice theorem, the slopes $\left(s_{1}, s_{2}\right)$ of an $x$-plane can be obtained by

$$
\begin{aligned}
\hat{R} I_{x}\left(s_{1}, s_{2}, k\right) & =\sum_{v, w=-n / 2}^{n / 2-1} \sum_{u=-n / 2}^{n / 2-1} I(u, v, w) e^{(-2 \pi i k / m)\left(u-s_{1} v-s_{2} w\right)}, \\
& =\hat{I}\left(k,-s_{1} k,-s_{2} k\right)
\end{aligned}
$$

where $k=\{-3 n / 2, \ldots, 3 n / 2\}$ and $\hat{I}$ is the trigonometric polynomial given by
$\hat{I}\left(\xi_{1}, \xi_{2}, \xi_{3}\right)=\sum_{u=-n / 2}^{n / 2-1} \sum_{v=-n / 2}^{n / 2-1} \sum_{w=-n / 2}^{n / 2-1} I(u, v, w) e^{(-2 \pi i / m)\left(\xi_{1} u+\xi_{2} v+\xi_{3} u\right)}$

The 3D Fourier slice theorem for the $x$-planes in equation A-7 says that the 1D Fourier transform of the Radon transform for a fixed direction $\left(s_{1}, s_{2}\right)$ is equal to the samples of $\hat{I}$ along the line whose direction vector is $\left(1,-s_{1},-s_{2}\right)$ at $\left(k,-s_{1} k,-s_{2} k\right)$, $k=-3 n / 2, \ldots, 3 n / 2$.

Similarly, the slopes $\left(s_{1}, s_{2}\right)$ of the $y$ - and z-planes can be obtained by

$$
\begin{aligned}
\hat{R} I_{y}\left(s_{1}, s_{2}, k\right) & =\sum_{u, w=-n / 2}^{n / 2-1} \sum_{v=-n / 2}^{n / 2-1} I(u, v, w) e^{(-2 \pi i k / m)\left(v-s_{1} u-s_{2} w\right)} \\
& =\hat{I}\left(-s_{1} k, k,-s_{2} k\right), \\
\hat{R} I_{z}\left(s_{1}, s_{2}, k\right) & =\sum_{u, v=-n / 2} \sum_{w=-n / 2}^{n / 2-1} I(u, v, w) e^{(-2 \pi i k / m)\left(w-s_{1} u-s_{2} v\right)} \\
& =\hat{I}\left(-s_{1} k,-s_{2} k, k\right) .
\end{aligned}
$$

In equations $\mathrm{A}-1, \mathrm{~A}-4$, and $\mathrm{A}-5$, the Radon transform operators $R_{1} I, R_{2} I$, and $R_{3} I$ are defined for a discrete image $I$ with continuous slopes $\left(s_{1}, s_{2}\right)$ in $[-1,1] \times[-1,1] ; s_{1}$ and $s_{2}$ can be discretized with the condition $\left|s_{1}\right| \leq 1$ and $\left|s_{2}\right| \leq 1$ by defining the sets $S_{1}$ and $S_{2}$ as

$$
\begin{aligned}
& S_{1} \triangleq\left\{\frac{l}{n / 2} \mid l=-\frac{n}{2}, \ldots, \frac{n}{2}\right\}, \\
& S_{2} \triangleq\left\{\frac{j}{n / 2} \mid j=-\frac{n}{2}, \ldots, \frac{n}{2}\right\},
\end{aligned}
$$

where the set of slopes $\left(s_{1}, s_{2}\right) \in S_{1} \times S_{2}$.

During the preconditioning, the local Radon transform coefficients are brought back to the image domain using the inverse Radon operator $\mathbf{R}^{-1}$, which is approximated by the adjoint operator $\mathbf{R}^{T}$ of the forward Radon transform operator $\mathbf{R}$. This is done because for large-scale problems, taking the adjoint is more efficient and more stable than taking the exact inverse. The adjoint operator $\mathbf{R}^{T}$ is constructed by taking the numerical adjoint of the forward transform steps described above. Beylkin (1987) proposes an exact inversion algorithm for the discrete Radon transform by casting the forward transform as a matrix-vector multiplication, in which the block-circulant nature of the transform matrix $\mathbf{R}$ allows for the construction of a fast inverse transform. The inversion approach proposed by Beylkin (1987) can also be adapted for the Fourier-based discrete Radon transform approach shown above. However, as it will be shown in our numerical tests, we did not see any problem because of using the adjoint instead of the inverse of the Radon operator.

The resolution of the local-Radon transform operator and the sparsity of the transform coefficients in Figure $1 \mathrm{~b}$ and $1 \mathrm{~d}$ can also be improved by using a model reweighted least-squares procedure as proposed by Sacchi and Ulrych (1995) and Trad et al. (2003). The images from the proposed LSRTM formulation using such a sparse Radon transform formulation are then expected to be better. 


\section{REFERENCES}

Anagaw, A. Y., and M. D. Sacchi, 2012, Edge-preserving seismic imaging using the total variation method: Journal of Geophysics and Engineering, 9, 138-146, doi: 10.1088/1742-2132/9/2/138.

Averbuch, A., and Y. Shkolnisky, 2003, 3D Fourier based discrete Radon transform: Applied and Computational Harmonic Analysis, 15, 33-69, doi: 10.1016/S1063-5203(03)00030-7.

Beylkin, G., 1987, Discrete Radon transform: IEEE Transactions on Acoustics, Speech, and Signal Processing, 35, 162-172, doi: 10.1109/TASSP .1987 .1165108

Boonyasiriwat, C., and G. Schuster, 2010, 3D multisource full-waveform inversion using dynamic quasi-Monte Carlo phase encoding: EGU General Assembly Conference Abstracts, 12, 7298.

Cabrales-Vargas, A., and K. J. Marfurt, 2013, Amplitude-preserving imaging of aliased data using preconditioned Kirchhoff least-squares depth migration: 83rd Annual International Meeting, SEG, Expanded Abstracts, 3726-3730.

Calvetti, D., S. Morigi, L. Reichel, and F. Sgallari, 2000, Tikhonov regularization and the 1-curve for large discrete ill-posed problems: Journal of Computational and Applied Mathematics, 123, 423-446, doi: 10 .1016/S0377-0427(00)00414-3.

Candes, E., L. Demanet, D. Donoho, and L. Ying, 2006a, Fast discrete curvelet transforms: Multiscale Modeling \& Simulation, 5, 861-899, doi: 10 $.1137 / 05064182 X$.

Candes, E. J., J. K. Romberg, and T. Tao, 2006b, Stable signal recovery from incomplete and inaccurate measurements: Communications on Pure and Applied Mathematics, 59, 1207-1223, doi: 10.1002/(ISSN)1097-0312.

Dai, W., C. Boonyasiriwat, and G. T. Schuster, 2010, 3D multi-source leastsquares reverse time migration: 80th Annual International Meeting, SEG, Expanded Abstracts, 3120-3124, doi: 10.1190/SEGEAB.29.

Dai, W., P. Fowler, and G. T. Schuster, 2012, Multisource least-squares reverse time migration: Geophysical Prospecting, 60, 681-695, doi: 10 .1111/gpr.2012.60.issue-4.

Dai, W., and G. T. Schuster, 2010, Multi-source wave equation least-squares migration with a deblurring filter: 72nd Annual International Conference and Exhibition, EAGE, Extended Abstracts, 276.

Dai, W., and G. T. Schuster, 2013, Plane-wave least-squares reverse-time migration: Geophysics, 78, no. 4, S165-S177, doi: 10.1190/geo2012-0377.1.

Daubechies, I., R. DeVore, M. Fornasier, and C. S. Güntürk, 2010, Iteratively reweighted least squares minimization for sparse recovery: Communications on Pure and Applied Mathematics, 63, 1-38, doi: 10 .1002/cpa.v63:1.

Duprat, V., and R. Baina, 2016, An efficient least-squares reverse-time migration using true-amplitude imaging condition as an optimal preconditioner: 78th Annual International Conference and Exhibition, EAGE Extended Abstracts, We SRS2 12

Duquet, B., K. J. Marfurt, and J. A. Dellinger, 2000, Kirchhoff modeling, inversion for reflectivity, and subsurface illumination: Geophysics, $\mathbf{6 5}$, 1195-1209, doi: $10.1190 / 1.1444812$.

Dutta, G., 2015, Sparse least-squares reverse time migration using seislets: 85th Annual International Meeting, SEG, Expanded Abstracts, 42324237.

Dutta, G., 2017, Sparse least-squares reverse time migration using seislets: Journal of Applied Geophysics, 136, 142-155.

Dutta, G., and G. T. Schuster, 2014, Attenuation compensation for least squares reverse time migration using the viscoacoustic-wave equation: Geophysics, 79, no. 6, S251-S262, doi: 10.1190/geo2013-0414.1.

Dutta, G., Y. Huang, W. Dai, X. Wang, and G. T. Schuster, 2014, Making the most out of the least (squares migration): 84th Annual International Meeting, SEG, Expanded Abstracts, 4405-4410.

Fomel, S., and A. Guitton, 2006, Regularizing seismic inverse problems by model reparameterization using plane-wave construction: Geophysics, $\mathbf{7 1}$ no. 5, A43-A47, doi: 10.1190/1.2335609.

Fomel, S., and Y. Liu, 2010, Seislet transform and seislet frame: Geophysics, 75, no. 3, V25-V38, doi: 10.1190/1.3380591.

Harlan, W. S., 1995, Regularization by model reparameterization, http:// www.billharlan.com/pub/papers/regularization.pdf, accessed 22 June 1997.
Herrmann, F., C. Brown, Y. Erlangga, and P. Moghaddam, 2009, Curveletbased migration preconditioning and scaling: Geophysics, 74, no. 4, A41A46, doi: 10.1190/1.3124753.

Herrmann, F. J., and X. Li, 2012, Efficient least-squares imaging with sparsity promotion and compressive sensing: Geophysical Prospecting, 60, 696-712, doi: 10.1111/gpr.2012.60.issue-4.

Herrmann, F. J., P. Moghaddam, and C. C. Stolk, 2008, Sparsity- and continuity-promoting seismic image recovery with curvelet frames: Applied and Computational Harmonic Analysis, 24, 150-173, doi: 10.1016/j.acha 2007.06.007.

Holland, P. W., and R. E. Welsch, 1977, Robust regression using iteratively reweighted least-squares: Communications in Statistics - Theory and Methods, 6, 813-827, doi: 10.1080/03610927708827533.

Ibrahim, A., and M. D. Sacchi, 2014, Simultaneous source separation using a robust Radon transform: Geophysics, 79, no. 1, V1-V11, doi: 10.1190/ geo2013-0168.1.

Kingsbury, N., 2001, Complex wavelets for shift invariant analysis and filtering of signals: Applied and Computational Harmonic Analysis, 10, 234-253, doi: 10.1006/acha.2000.0343.

Lailly, P., 1984, Migration methods: partial but efficient solutions to the seismic inverse problem: Inverse Problems of Acoustic and Elastic Waves, 51, $1387-1403$

Li, C., J. Huang, Z. Li, and R. Wang, 2015, Regularized least-squares reverse time migration with prior model: 85th Annual International Meeting, SEG, Expanded Abstracts, 4143-4147.

Lin, Y., and L. Lianjie, 2015, Least-squares reverse-time migration with modified total-variation regularization: 85th Annual International Meeting, SEG, Expanded Abstracts, 4264-4269.

Miller, M. A., N. G. Kingsbury, and R. W. Hobbs, 2005, Seismic image reconstruction using complex wavelets: Electronic Imaging, 5674, 27-35.

Morton, S. A., and C. C. Ober, 1998, Faster shot record depth migrations using phase encoding: 68th Annual International Meeting, SEG, Expanded Abstracts, 1131-1134.

Nemeth, T., C. Wu, and G. T. Schuster, 1999, Least-squares migration of incomplete reflection data: Geophysics, 64, 208-221, doi: 10.1190/1 .1444517 .

Nocedal, J., and S. Wright, 1999, Numerical optimization: Springer Verlag.

Plessix, R.-E., and W. A. Mulder, 2004, Frequency-domain finite-difference amplitude-preserving migration: Geophysical Journal International, 157, 975-987, doi: 10.1111/gji.2004.157.issue-3.

Romero, L. A., D. C. Ghiglia, C. C. Ober, and S. A. Morton, 2000, Phase encoding of shot records in prestack migration: Geophysics, 65, 426-436, doi: $10.1190 / 1.1444737$.

Saad, Y., 2003, Iterative methods for sparse linear systems: SIAM.

Sacchi, M. D., and T. J. Ulrych, 1995, Improving resolution of Radon operators using a model re-weighted least squares procedure: Journal of Seismic Exploration, 4, 315-328.

Scales, J. A., A. Gersztenkorn, and S. Treitel, 1988, Fast Lp solution of large, sparse, linear systems: Application to seismic travel time tomography: Journal of Computational Physics, 75, 314-333, doi: 10.1016/ 0021-9991(88)90115-5.

Schuster, G. T., 1993, Least-squares cross-well migration: 63rd Annual International Meeting, SEG, Expanded Abstracts, 110-113.

Schuster, G. T., X. Wang, Y. Huang, W. Dai, and C. Boonyasiriwat, 2011, Theory of multisource crosstalk reduction by phase-encoded statics: Geophysical Journal International, 184, 1289-1303, doi: 10.1111/gji.2011 .184.issue-3.

Tang, Y., 2009, Target-oriented wave-equation least-squares migration/inversion with phase-encoded Hessian: Geophysics, 74, no. 6, WCA95WCA107, doi: 10.1190/1.3204768.

Trad, D., T. Ulrych, and M. Sacchi, 2003, Latest views of the sparse Radon transform: Geophysics, 68, 386-399, doi: 10.1190/1.1543224.

Wang, J., and M. D. Sacchi, 2007, High-resolution wave-equation amplitude-variation-with-ray-parameter (AVP) imaging with sparseness constraints: Geophysics, 72, no. 1, S11-S18, doi: 10.1190/1 .2387139 .

Wong, M., S. Ronen, and B. Biondi, 2011, Least-squares reverse time migration/inversion for ocean bottom data: A case study: 81st Annual International Meeting, SEG, Expanded Abstracts, 2369-2373. 